\title{
Health-Related Quality of Life in Women with Eating Disorders: Association with Subjective and Objective Binge Eating
}

\author{
Janet D. Latner · Joanna K. Vallance • \\ Geoffrey Buckett
}

Published online: 30 April 2008

(C) Springer Science+Business Media, LLC 2008

\begin{abstract}
This study examined health-related quality of life (QOL) and its association with different forms of binge eating in 53 women with eating disorders. Participants had enrolled in treatment for anorexia nervosa, bulimia nervosa, binge eating disorder, or other eating disorders not otherwise specified and completed measures of QOL, eating-related psychopathology, and mood disturbance. Eating- and mood-related psychopathology, and to a lesser extent, mental-component QOL scores, were severely impaired in this sample relative to population norms. QOL was significantly and independently predicted by subjective bulimic episodes and compensatory behaviors, including food avoidance, laxative abuse, and self-induced vomiting, accounting for $32 \%$ of the variance. Subjective bulimic episodes and food avoidance also independently predicted the physical-component QOL, accounting for $27 \%$ of the variance. These findings suggest that subjective bulimic episodes may be independently associated with impairment in QOL and may require specific attention as targets of treatment.
\end{abstract}

Keywords Eating disorders - Binge eating .

Subjective bulimic episodes - Objective bulimic episodes . Quality of life

\author{
J. D. Latner ( $\square)$ \\ Department of Psychology, University of Hawaii at Manoa, 2430 \\ Campus Road, Honolulu, HI 96822, USA \\ e-mail: jlatner@hawaii.edu \\ J. K. Vallance \\ Department of Psychology, University of Canterbury, \\ Christchurch, New Zealand \\ G. Buckett \\ Princess Margaret Hospital, Christchurch, New Zealand
}

Binge eating is a core symptom of the eating disorders. Binge eating is defined as the intake of a large amount of food accompanied by a loss of control over eating, also known as objective bulimic episodes (OBEs; Fairburn \& Cooper, 1993). However, it has been demonstrated that episodes of smaller food intake can also be accompanied by a loss of control over eating. These episodes, termed subjective bulimic episodes (SBEs; Fairburn \& Cooper, 1993), are often considered binge eating episodes by patients (Telch, Pratt, \& Niego, 1998) and are clinically significant. For example, among community women with a range of eating disorder symptoms, SBEs were as closely associated with eating-related and general psychopathology as were OBEs (Latner, Hildebrandt, Rosewall, Chisholm, \& Hayashi, 2007). Among 5,232 community women, SBE frequency was associated with significant functional impairment and SBEs were particularly impairing when found in combination with compensatory behaviors (e.g., self-induced vomiting, laxative abuse; Mond et al., 2006c).

In bulimia nervosa and binge eating disorder patients, SBEs have been shown to be difficult to treat, responding more slowly to treatment than OBEs (Niego, Pratt, \& Agras, 1997), not responding to early treatment strategies as well as OBEs do (Hildebrandt \& Latner, 2006), and persisting after treatment ends (Loeb, Wilson, Gilbert, \& Labouvie, 2000; Walsh, Fairburn, Mickley, Sysko, \& Parides, 2004). The difficulty in treating SBEs and their link with clinical impairment suggest that SBEs may require specific attention in treatment.

Previous research has established that health-related QOL can be useful as a measure of the general impairment associated with eating disorders (Doll, Petersen, \& Stewart- Brown, 2005; Hay \& Mond, 2005) as well as a measure of the impairment associated with particular symptoms of eating disorders. Additionally, in non-clinical and some clinical 
populations, certain eating-related symptoms have been shown to be associated with impairment in QOL, such as excessive exercise, SBEs (Mond, Hay, Rodgers, \& Owen, 2006a, b), and purging (Gonzalez-Pinto et al., 2004). Thus, the purpose of the present study was to measure QOL in a sample of women with eating disorders and to assess the relative contribution to QOL of the two different forms of binge eating, OBEs and SBEs. It was predicted that binge eating in one or both forms would significantly contribute to QOL.

\section{Method}

The study design and procedures used were approved by the Upper South Ethics Committee of the New Zealand Ministry of Health, and informed consent was obtained from participants.

\section{Participants}

Participants were recruited from all patients currently in outpatient treatment, on the waiting list for treatment, or discharged from treatment in the past several months from the Eating Disorders Service at the Princess Margaret Hospital, a general medical hospital in Christchurch, New Zealand. Fifty-three women consented to participate and returned their questionnaires. The majority of participants $(62 \%)$ were currently in treatment, $11 \%$ were on the waiting list, and $26 \%$ were recently discharged.

\section{Procedures}

Patients were offered the opportunity to complete study questionnaires on their own time. All information was obtained from participants (clinic files were not accessed). The battery of self-report questionnaires included the following measures, along with questions about demographic information and treatment status.

\section{Eating Disorders Examination-Questionnaire}

(EDE-Q; Fairburn \& Beglin, 1994). The EDE-Q is a 36item self-report version of the Eating Disorder Examination interview (Fairburn \& Cooper, 1993). The EDE-Q assesses the presence of diagnostic symptoms, as well as core eating disorder psychopathology in four domains: restraint, shape concerns, weight concerns, and eating concerns, focusing on the past 28 days. Attitudinal ratings are made on a 7-point scale, with higher scores indicating greater severity. (Sample item: "How dissatisfied have you felt about your weight?")

The EDE-Q has shown good internal consistency and good concurrent and criterion validity, and good correspondence of subscale ratings between the EDE-Q and EDE (Black \& Wilson, 1996; Carter, Aime, \& Mills, 2001; Fairburn \& Beglin, 1994; Mond, Hay, Rodgers, Owen \& Beumont, 2004). Studies have shown that for the assessment of OBEs, the EDE-Q is correlated with the EDE (Fairburn \& Beglin, 1994; Grilo, Masheb, \& Wilson, 2001) although some studies have shown higher OBE frequencies on the EDE (Carter et al., 2001; Mond et al., 2004; Wilfley, Schwartz, Spurrell, \& Fairburn, 1997) and some have shown higher frequencies on the EDE-Q (Black \& Wilson, 1996; Fairburn \& Beglin, 1994). Results are mixed regarding the correspondence between EDE and EDE-Q frequencies of SBEs, with some investigations finding significant correlations (Carter et al., 2001; Mond et al., 2004) and others showing insignificant correlations (Grilo et al., 2001). Although in cases of low correspondence it is generally assumed that the EDE is more accurate than the EDE-Q, it may be that the EDE is problematic in its reliability for SBE assessment (Grilo, Lozano, \& Elder, 2005; Grilo, Masheb, Lozano-Blanco, \& Barry, 2004), which might relate to difficulties inherent in the assessment of binge eating (Wilfley et al., 1997).

\section{The Medical Outcomes Short-form Health Survey}

(SF-36; Ware \& Sherbourne, 1992). The SF-36 is a 36-item measure of daily functioning. It assesses eight dimensions of generic health-related QOL, including physical functioning, role-physical, bodily pain, general health, vitality, social functioning, mental health, and role-emotional. The physical component summary (PCS), consisting of the first four of these domains, and the mental component summary (MCS), consisting of the last four, are global measures of physical and mental QOL, respectively. The total score represents an overall measure of QOL. (Sample item: "During the past 4 weeks, how much of the time has your physical health or emotional problems interfered with your social activities (like visiting with friends, relatives etc.)?") Lower scores indicate greater physical, social, and self-care limitations, poorer general health, greater psychological distress, and limitations in social activities due to emotional problems (Ware, Kosinski, \& Keller, 1994). The overall scale and summary scores have been demonstrated to be valid and reliable measures of QOL (Ware et al., 1994) and useful in assessing the health impairments of patients with eating disorders (Doll et al., 2005; Hay, 2003).

\section{Beck Depression Inventory II}

(BDI-II; Beck, Steer, Ball, \& Ranieri, 1996). The BDI-II is a 21-item measure of symptoms of depression during the past two weeks. Items are scored on a 4-point scale, with 
higher scores indicating greater depressive symptoms. (Sample item: " $0=$ I do not feel sad, $1=$ I feel sad much of the time, $2=\mathrm{I}$ am sad all the time, $3=\mathrm{I}$ am so sad or unhappy that I can't stand it.) The BDI-II has well-established reliability and validity, and it is widely used (Beck, Ward, Mendelson, Mock, \& Erbaugh, 1961; Sprinkle et al., 2002; Storch, Roberti, \& Roth, 2004). Total scores are generally considered to represent minimal symptoms (013), mild symptoms (14-19), moderate symptoms (20-28) or severe symptoms (29-63).

\section{Statistical Analysis}

The mean QOL and summary scores in the present study were computed and compared (descriptively) alongside normative data on QOL and EDE-Q scores. To assess the relative contributions of the two forms of binge eating to QOL, OBEs and SBEs were entered into three multiple regression analyses, with overall SF-36 scores, PCS, and MCS scores as the dependent variable in each analysis. To control for the contributions of compensatory behaviors designed to influence shape and weight, frequencies of these behaviors were also included as potential predictors in the model. These included self-induced vomiting, laxative abuse, excessive exercise, and food avoidance, defined by the EDE-Q question concerning fasting for a period of 8 or more hours. In addition, regression analysis examined these forms of binge eating and compensatory behaviors as potential predictors of BDI-II scores, as a measure of general psychopathology (mood disturbance). Significance level (alpha) was set at $p<.05$.

\section{Results}

\section{Participant Characteristics}

Participants' mean age (SD) was 26.30 (8.98) years, and their mean current body mass index $\left(\mathrm{BMI} ; \mathrm{kg} / \mathrm{m}^{2}\right.$ ) was 21.58 (7.28). Ninety-four percent of participants were of European decent, $2 \%$ were Asian, 2\% were Maori, and 2\% were Pacific Islander. The majority of participants (64\%) had never been married, $13 \%$ were separated, $6 \%$ were divorced, $6 \%$ were widowed, and $8 \%$ were cohabitating, and 3\% did not indicate their marital status. Currently 34\% were students, $36 \%$ were professionally employed, $17 \%$ were homemaker/mothers, $8 \%$ were disability benefit recipients, $2 \%$ were unemployed, and $2 \%$ did not indicate their occupational status.

Among those patients who had been diagnosed by clinicians at the Eating Disorders Service, 49\% reported having received a diagnosis (when starting treatment) of anorexia nervosa (AN), $8 \%$ of bulimia nervosa (BN), $2 \%$ of binge eating disorder (BED), $19 \%$ of other eating disorders not otherwise specified (EDNOS). The remaining 22\% had either not yet been diagnosed or did not report their diagnoses. Based on responses to the EDE-Q and demographic questionnaire at the time of the present investigation, participants fit into the following DSM-IV (American Psychiatric Association; APA, 2000) diagnostic categories: $21 \%$ met criteria for AN; $9 \%$ BN; $6 \%$ BED; $56 \%$ other EDNOS (this included $25 \%$ of the total sample who were close to meeting criteria for AN but were just above a BMI of 17.5), and $8 \%$ no longer met criteria for an eating disorder. There were no differences in overall QOL (total SF36 scores, PCS, or MCS) across diagnostic groups, as revealed by either ANOVA or $t$-tests comparing the two largest groups, AN and EDNOS. This was true for originally assigned diagnoses as well as current diagnoses based on EDE-Q and demographic responses. There were also no differences in QOL between patients currently in treatment, on the waiting list, or recently discharged from treatment.

\section{QOL Impairment}

Participants mean QOL scores and EDE-Q scores are shown in Table 1, alongside normative scores from New Zealand (Scott, Tobias, Sarfati, \& Haslett, 1999) and Australian (Mond et al., 2006b; Mishra \& Schofield, 1998) populations of similar aged women. These descriptive comparisons suggest that the PCS scores in the present sample were comparable to population scores, while MCS scores were somewhat lower than population scores. The frequency of binge eating and compensatory behaviors is also shown in Table 1.

The mean BDI-II score (SD) in the sample was 24.60 (15.49), comparable to a mean of 22.36 (11.92) in a group of psychiatric outpatients with mood, anxiety, and other disorders (Beck et al., 1996). Among 569 New Zealand non-clinical women, the mean score was 2.82 (3.54) on the short-form BDI (Knight, 1984). Converting this normative score into a comparable 21-item scale score (by dividing this sum by 13 and multiplying by 21) yields a mean of 4.56 , indicating over a five-fold increase in the present sample.

\section{Contributions of Binge Eating and Compensatory} Behavior to Impairment

Regression analyses examined the simultaneous relative contributions to QOL of the two forms of binge eating, OBEs and SBEs, alongside compensatory behaviors. (Diuretic misuse was present in less than $4 \%$ of patients and thus was not entered into the model.) Total SF-36 scores were significantly predicted by this regression model 
Table 1 Means (SD) of QOL and EDE-Q scores in the present sample, and means (SD) among Australian and New Zealand non-clinical populations (where available)

a Mishra and Schofield (1998) $(N=14,780$ women aged $18-$ 22 years)

b Scott et al. (1999) $(N=1883$ women aged 25-44)

${ }^{c}$ Mond et al. (2006a, b) $(N=908$ women aged 23-27)

\begin{tabular}{lll}
\hline & Present sample: Means (SD) & Population norms: Means (SD) \\
\hline SF-36 PCS & $53.14(4.91)$ & $48.89(8.70)^{\mathrm{a}}$ \\
& & $52.30(13.02)^{\mathrm{b}}$ \\
SF-36 MCS & $42.99(4.61)$ & $45.35(12.46)^{\mathrm{a}}$ \\
& & $47.70(13.02)^{\mathrm{b}}$ \\
SF-36 Total & $96.13(6.92)$ & $94.24^{\mathrm{a}}$ \\
& & $100.0^{\mathrm{b}}$ \\
EDE-Q Restraint & $3.37(1.86)$ & $1.34(1.39)^{\mathrm{c}}$ \\
EDE-Q Eating Concern & $2.77(1.56)$ & $0.81(1.10)^{\mathrm{c}}$ \\
EDE-Q Shape Concern & $4.45(1.69)$ & $2.24(1.61)^{\mathrm{c}}$ \\
EDE-Q Weight Concern & $4.14(1.70)$ & $1.84(1.50)^{\mathrm{c}}$ \\
EDE-Q Global & $3.68(1.53)$ & $1.56(1.26)^{\mathrm{c}}$ \\
OBE Frequency & $4.31(6.78)$ & - \\
SBE Frequency & $9.33(9.42)$ & - \\
Self-induced vomiting frequency & $9.64(21.98)$ & - \\
Laxative abuse frequency & $1.34(4.84)$ & - \\
Food avoidance frequency & $1.85(2.30)$ & - \\
Excessive exercise frequency & $6.35(8.86)$ & - \\
Diuretic use frequency & $0.23(1.40)$ & \\
\hline
\end{tabular}

$(F(6,42)=3.28, p<.01)$, which accounted for $32 \%$ of the variance. SBEs $(\beta=.41, \quad p<.01)$, food avoidance $(\beta=.40, p<.05)$, laxative abuse $(\beta=.31, p<.05)$, and self-induced vomiting $(\beta=.29, p<.05)$ had independently significant beta values. Neither OBEs nor excessive exercise were independent predictors of overall QOL.

Similar results emerged in a regression model predicting PCS scores $(F(6,42)=2.59, p<.05)$, which accounted for $27 \%$ of the variance. SBEs $(\beta=.38, p<.05)$ and food avoidance $(\beta=.37, p<.05)$ were independently significant predictors, whereas OBEs, self-induced vomiting, laxative abuse, and excessive exercise were not. The regression analysis predicting MCS scores was not significant $(F=0.77$, n.s.).

A final regression analysis was conducted to examine predictors of BDI-II scores, and this model accounted for $29 \%$ of the variance $(F(6,42)=4.19, p<.005)$. Independently significant predictors included only laxative abuse $(\beta=.32, p<.05)$ and food avoidance $(\beta=.31, p<.05)$. For each of these analyses, collinearity diagnostics (Eigenvalues and condition indices) revealed no problems with multi-collinearity.

\section{Discussion}

In the current sample the frequency of subjective bulimic episodes (SBEs) significantly contributed to both general and physical QOL. Specific compensatory behaviors also significantly contributed to QOL, including food avoidance, laxative abuse, and self-induced vomiting. However, objective bulimic episodes (OBEs) did not independently predict overall, physical, or mental QOL. In predicting symptoms of depression, only compensatory behaviors (laxative abuse and food avoidance) were independently significant.

It can often be difficult to independently evaluate the effects or correlates of OBEs and SBEs as they tend to cooccur (e.g., Mond et al., 2006c). In this study, however, the correlation between these two forms of binge eating was not significant ( $r=.21$, n.s.), suggesting a relatively low degree of overlap and permitting an evaluation of their independent effects.

This sample showed a high level of eating-related and general psychopathology, and somewhat lower mental QOL than the general population. This decreased mental QOL may indicate interference with normal social activities, frequent feelings of nervousness and depression, problems with work or daily activities, and/or feeling worn out or tired due to emotional or physical health problems (Ware \& Sherbourne, 1992). However, the physical and overall QOL in this sample were not dramatically worse than population norms. This may seem surprising and differs from some previous research showing substantial impairments in QOL among eating disordered women. However, the present results are also similar to prior findings of unexpectedly high reported QOL with AN patients (Mond, Hay, Rodgers, Owen, \& Beumont, 2005; Padierna, Quintana, Arostegui, Gonzalez, \& Horcajo, 2000).

It is possible that the self-report of similar QOL of our sample to normative samples stems from the ego-syntonic 
aspects of eating disorders and denial of its consequences (Vitousek, Watson, \& Wilson, 1998). For example, at the Eating Disorders Service at Princess Margaret Hospital, a great deal of time in treatment is spent with patients and their families in an effort to increase their understanding that they are indeed ill. This can be especially difficult among athletes, who may feel that their low weight or amenorrhea are performance enhancing and, therefore, positive. The current findings that bulimic episodes predict lower mental QOL are consistent with the clinical observation that for many patients, bulimic episodes stand out as the most ego-dystonic feature of eating disorders overall. Some patients may be willing to acknowledge physical disturbances such as global tiredness, coldness and other effects of profound starvation. However, these symptoms are typically experienced by severely underweight patients, while the present sample had a mean BMI of 21.6. In addition, the physical symptoms of bulimia nervosa can include hypokalemia, cardiac and dental problems, but these specific conditions are not likely to be captured by the SF-36. Finally, the physical symptoms of eating disorders often compound and increase over the years, but the present sample (mean age $=26.3$ ) may not be old enough to have developed the problems of chronic eating disorders. Later in life, patients who are still suffering from these disorders may have very significant reductions in physical QOL.

It is also possible that the similar physical and overall QOL scores of the present sample to a normative population of 18-22 year old women (Mishra \& Schofield, 1998) may result from impairment in the normative sample. The frequency of body image dissatisfaction, dieting, and drive to be thin in young women may have an impact on both the present sample and the normative group, whose QOL scores were lower than an older normative sample with a broader age range (Scott et al., 1999), as shown in Table 1.

There were several limitations of the present study. First, the sample was not uniform with regard to diagnostic category or treatment status; however, this variability in the sample's symptom levels may also have served as an advantage by permitting us to examine the effects of higher and lower levels of binge eating on QOL. The self-reported assessment of eating disorder symptoms was also a limitation of the study. Some studies have shown low correspondence between questionnaire and interview measures for the assessment of binge eating, especially SBEs (Grilo et al., 2001), and this is usually attributed to problems with the questionnaire measures. However, some studies have shown correspondence between questionnaire and interview measures (Carter et al., 2001; Mond et al., 2004). Indeed, even the supposedly best available measure for assessing SBEs, the EDE interview, is also flawed in this respect, with poor test-retest reliability (Grilo et al.,
2004, 2005). This suggests that improved measures to assess SBEs are critically needed. Future research should focus on improving the assessment of SBEs through more psychometrically sophisticated, reliable, and valid measures. In addition, future research should further examine quality of life among community-based samples of individuals with eating disorders (Hay \& Mond, 2005).

The present findings may have important implications for diagnosis and clinical practice. Despite the clinical significance of SBEs, they are not included as a diagnostic criterion for any DSM-IV eating disorder (APA, 2000). In the development of the next version of the DSM (DSM-V), it may be beneficial to consider the growing body of evidence on the clinical implications of this symptom (Latner \& Clyne, 2008). The present findings also suggest that SBEs, as contributors to psychopathology, may be important to include as specific targets in treatment. Specifically, clinicians should be aware of the importance of assessing, being sensitive to, and addressing client-perceived binges in the context of cognitive behavioral treatment. If practitioners define and address only what they consider objective bulimic episodes, they may overlook patients' distress about feeling out of control and overeating. This may lead patients to feel misunderstood, invalidated, or dissatisfied with treatment and its outcome. Their continued subjective loss of control and resulting distress may further erode patients' sense of self-efficacy, undermine their commitment to behavioral change, and adversely affect their compliance with behavioral assignments designed to enhance their functioning and quality of life.

\section{References}

American Psychiatric Association. (2000). Diagnostic and statistical manual of mental disorders (4th ed.). Washington, DC: Author.

Beck, A. T., Steer, R. A., Ball, R., \& Ranieri, W. F. (1996). Comparison of Beck Depression Inventories-IA and -II in psychiatric outpatients. Journal of Personality Assessment, 67, 588-597.

Beck, A. T., Ward, C. H., Mendelson, M., Mock, J., \& Erbaugh, J. (1961). An inventory for measuring depression. Archives of General Psychiatry 4, 561-571.

Black, C. M., \& Wilson, G. T. (1996). Assessment of eating disorders: Interview versus questionnaire. International Journal of Eating Disorders, 20, 43-50.

Carter, J. C., Aime, A. A., \& Mills, J. S. (2001). Assessment of bulimia nervosa: A comparison of interview and self-report questionnaire methods. International Journal of Eating Disorders, 30, 187-192.

Doll, H. A., Petersen, S. E., \& Stewart-Brown, S. L. (2005). Eating disorders and emotional and physical well-being: Associations between student self-reports of eating disorders and quality of life as measured by the SF-36. Quality of Life Research, 14, 705717.

Fairburn, C. G., \& Beglin, S. J. (1994). Assessment of eating disorders: Interview or self-report questionnaire? International Journal of Eating Disorders, 16, 363-370. 
Fairburn, C. G., \& Cooper, Z. (1993). The Eating Disorder Examination. In C. G. Fairburn \& G. T. Wilson (Eds.), Binge eating: Nature, assessment, and treatment (12th ed., pp. 317332). New York: Guilford Press.

Gonzalez-Pinto, A., Inmaculada, F., Cristina, R., Corres Blanca, F., Sonsoles, E., Fernando, R., et al. (2004). Purging behaviors and comorbidity as predictive factors of quality of life in anorexia nervosa. International Journal of Eating Disorders, 36, 445-450.

Grilo, C. M., Lozano, C., \& Elder, K. A. (2005). Inter-rater and testretest reliability of the spanish language version of the Eating Disorder Examination interview: Clinical and research implications. Journal of Psychiatric Practice, 11, 231-240.

Grilo, C. M., Masheb, R. M., Lozano-Blanco, C., \& Barry, D. T. (2004). Reliability of the Eating Disorder Examination in patients with binge eating disorder. International Journal of Eating Disorders, 35, 80-85.

Grilo, C. M., Masheb, R. M., \& Wilson, G. T. (2001). A comparison of different methods for assessing the features of eating disorders in patients with binge eating disorder. Journal of Consulting and Clinical Psychology, 69, 317-322.

Hay, P. (2003). Quality of life and bulimic eating disorder behaviors: Findings from a community-based sample. International Journal of Eating Disorders, 33, 434-442.

Hay, P., \& Mond, J. (2005). How to 'count the cost' and measure burden? A review of health-related quality of life in people with eating disorders. Journal of Mental Health, 14, 539-552.

Hildebrandt, T., \& Latner, J. D. (2006). Effect of self-monitoring on binge eating: Treatment response or "binge drift"? European Eating Disorders Review, 14, 16-22.

Knight, R. G. (1984). Some general population norms for the short form Beck Depression Inventory. Journal of Clinical Psychology, 40, 751-753.

Latner, J. D., \& Clyne, C. (2008). The diagnostic validity of the criteria for binge eating disorder. International Journal of Eating Disorders, 41, 1-14.

Latner, J. D., Hildebrandt, T., Rosewall, J. K., Chisholm, A. M., \& Hayashi, K. (2007). Loss of control over eating reflects eating disturbances and general psychopathology. Behaviour Research and Therapy, 45, 2203-2211.

Loeb, K. L., Wilson, G. T., Gilbert, J. S., \& Labouvie, E. (2000). Guided and unguided self-help for binge eating. Behaviour Research and Therapy, 38, 259-272.

Mishra, G., \& Schofield, M. J. (1998). Norms for the physical and mental health component summary scores of the SF-36 for young, middle-aged and older Australian women. Quality of Life Research, 7, 215-220.

Mond, J. M., Hay, P. J., Rodgers, B., Owen, C., \& Beumont, P. J. V. (2004). Validity of the Eating Disorder Examination Questionnaire (EDE-Q) in screening for eating disorders in community samples. Behaviour Research and Therapy, 42, 551-567.

Mond, J. M., Hay, P. J., Rodgers, B., Owen, C., \& Beumont, P. J. V. (2005). Assessing quality of life in eating disorder patients. Quality of Life Research, 14, 171-178.
Mond, J. M., Hay, P. J., Rodgers, B., \& Owen, C. (2006a). An update on the definition of "excessive exercise" in eating disorders research. International Journal of Eating Disorders, 39, 147153.

Mond, J. M., Hay, P. J., Rodgers, B., \& Owen, C. (2006b). Eating Disorder Examination Questionnaire (EDE-Q): Norms for young adult women. Behaviour Research and Therapy, 44, 53-62.

Mond, J., Hay, P., Rodgers, B., Owen, C., Crosby, R., \& Mitchell J. (2006c). Use of extreme weight control behaviors with and without binge eating in a community sample: Implications for the classification of bulimic-type eating disorders. International Journal of Eating Disorders, 39, 294-302.

Niego, S. H., Pratt, E. M., \& Agras, W. S. (1997). Subjective or objective binge: Is the distinction valid? International Journal of Eating Disorders, 22, 291-298.

Padierna, A., Quintana, J. M., Arostegui, I., Gonzalez, N., \& Horcajo, M. J. (2000). The health-related quality of life in eating disorders. Quality of Life Research, 9, 667-674.

Scott, K. M., Tobias, M. I., Sarfati, D., \& Haslett, S. J. (1999). SF-36 health survey reliability, validity and norms for New Zealand. Australian and New Zealand Journal of Public Health, 23, 401407.

Sprinkle, S. D., Lurie, D., Insko, S. L., Atkinson, G., Jones, G., Logan, A. R., et al. (2002). Criterion validity, severity cut scores, and test-retest reliability of the Beck Depression Inventory-II in a university counseling center sample. Journal of Counseling Psychology, 49, 381-385.

Storch, E. A., Roberti, J. W., \& Roth, D. A. (2004). Factor structure, concurrent validity, and internal consistency of the Beck Depression Inventory-second edition in a sample of college students. Depression and Anxiety, 19, 187-189.

Telch, C. F., Pratt, E. M., \& Niego, S. H. (1998). Obese women with binge eating disorder define the term binge. International Journal of Eating Disorders, 24, 313-317.

Vitousek, K., Watson, S., \& Wilson, G. T. (1998). Enhancing motivation for change in treatment-resistant eating disorders. Clinical Psychology Review, 18, 391-420.

Walsh, B. T., Fairburn, C. G., Mickley, D., Sysko, R., \& Parides, M. K. (2004). Treatment of bulimia nervosa in a primary care setting. American Journal of Psychiatry, 161, 556-561.

Ware, J. E., Kosinski, M., \& Keller, S. (1994). SF-36 physical and mental summary scales: A users manual. Boston, Massachusetts: The Health Institute, New England Medical Centre.

Ware, J., \& Sherbourne, C. (1992). The MOS 36-item Short-Form Health Survey (SF-36). Medical Care, 30, 473-483.

Wilfley, D. E., Schwartz, M. B., Spurrell, E. B., \& Fairburn, C. G. (1997). Assessing the specific psychopathology of binge eating disorder patients: Interview or self-report? Behaviour Research and Therapy, 35, 1151-1159. 